\title{
Prevalence, antibiotic susceptibility profiles and ESBL production in Klebsiella pneumoniae and Klebsiella oxytoca among hospitalized patients
}

\author{
SOURAV CHAKRABORTY1 \\ KANIZ MOHSINA ${ }^{1}$ \\ PALASH KUMAR SARKER ${ }^{2}$ \\ MD ZAHANGIR ALAM ${ }^{3}$ \\ M ISMAIL ABDUL KARIM ${ }^{3}$ \\ S M ABU SAYEM ${ }^{1,3 \S}$ \\ ${ }^{1}$ Department of Genetic Engineering \\ and Biotechnology, \\ Shahjalal University of Science and Technology, \\ Sylhet, Bangladesh \\ ${ }^{2}$ Microbial Biotechnology Division, \\ National Institute of Biotechnology, Savar, \\ Dhaka, Bangladesh \\ ${ }^{3}$ Department of Biotechnology Engineering, \\ International Islamic University of Malaysia, \\ Kuala Lumpur, Malaysia \\ Correspondence: \\ S M Abu Sayem \\ Email: asayem08@yahoo.com
}

Keywords: Multi-drug resistant, Klebsiella, extendedspectrum beta-lactamase.

Received April 30, 2015.

Revised January 25, 2016

Accepted January 25, 2016

\begin{abstract}
Background and Purpose: Klebsiella pneumoniae and Klebsiella oxytoca are the two most common pathogens causing nosocomial infections in humans and are of great concern for developing multidrug resistance. In the present study, K. pneumoniae and K. oxytoca from clinical samples were evaluated for their antibiotic sensitivity patterns against commonly used antibiotics and production of extended-spectrum beta-lactamase (ESBL).
\end{abstract}

Materials and Methods: The isolates were obtained from tracheal swabs, sputum, wound swabs, pus, blood and urine samples of hospitalized patients. Klebsiella pneumoniae and Klebsiella oxytoca were identified by cultural and biochemical methods. Antibiotic sensitivity test was performed by modified Kirby-Bauer disc diffusion technique. ESBL production in Klebsiella spp. was confirmed by double disc synergy test.

Results and Conclusion: Out of 500 clinical isolates, 120 were found positive for Klebsiella among which 108 were K. pneumoniae and 12 were K. oxytoca based on indole test. Prevalence rate of Klebsiella was found more prominent in males aged over 50 years, mostly in urine samples. Overall resistance pattern of Klebsiella isolates to Ampicillin, Amoxicillin, Ceftriaxone, Ciprofloxacin, Co-trimoxazole, Gentamicin, Nalidixic acid, Tetracycline was 100\%, 90\%, 45\%, 40\%, 45\%, 25\%, 50\%, 35\% respectively. Multidrug resistance was found more common in $\mathrm{K}$. pneumoniae (56\%) than in K. oxytoca (50\%). Prevalence rate of ESBL producing Klebsiella was found $45 \%$ among which $\mathrm{K}$. pneumoniae (50\%) were found more prominent than K. oxytoca (25\%). All the ESBL producing Klebsiella isolates were found to be multidrug resistant, showing $100 \%$ resistance to Ampicillin, Amoxicillin, Ceftriaxone and Ciprofloxacin.

\section{INTRODUCTION}

Tlebsiella have been considered as one of the major opportunistic 1 pathogens that cause a range of clinical diseases including nosocomial pneumonia, urinary tract infections and bacteremia in immunocompromised humans (8). Among the five gram-negative pathogens for hospital-acquired infections, Klebsiella is the most commonly encountered pathogen (14). The leading Klebsiella species giving rise to infections in humans are Klebsiella pneumoniae and Klebsiella oxytoca (13). K. pneumoniae accounts for $75 \%$ to $86 \%$ of all Klebsiella species reported while Klebsiella oxytoca accounts for $13 \%$ to $25 \%$ of isolates $(12,38)$. 
Emergence of antimicrobial resistance among Klebsiella species is becoming a global public health concern. High rate of antibiotic resistance is an inevitable consequence of widespread use of antibiotics in medical practice in developing countries $(28,30)$. In addition, resistance to broad spectrum beta-lactam antibiotics via extended-spectrum beta-lactamase (ESBL) production is an increasing problem worldwide (24). Klebsiella and E. coli were reported to be the most common pathogenic microorganisms to develop resistance to broad spectrum beta-lactam antibiotics via extended-spectrum beta-lactamase (ESBL) (2). Several risk factors for ESBL-producing Klebsiella spp. infections have been described for the most frequent antimicrobial exposure, especially to thirdgeneration cephalosporins $(18,25)$ which resulted in increased morbidity, mortality and costs of health care. Failure in the treatment of infectious diseases especially caused by Klebsiella, therefore, need to be under controlled monitoring in developing countries like Bangladesh to avoid widespread distribution of multidrug resistant Klebsiella spp.

Therefore, the present study aims to evaluate the prevalence of $K$. pneumonia and $K$. oxytoca among clinical patients and to analyze ESBL production and antimicrobial susceptibility pattern against most commonly prescribed antibiotics in order to treat patients with diseases caused by $K$. pneumonia and $K$. oxytoca.

\section{MATERIALS AND METHODS}

\section{Sample collection and processing}

A total of 500 clinical samples including blood, urine, pus and sputum were obtained from Popular Diagnostic Centre and Medinova Diagnostic Centre of Sylhet branch, Bangladesh from November 2013 to October 2014. The patients were of different ages and also of both sexes. The collected samples were then collected and processed according to standard operating conditions.

\section{Isolation and identification of Klebsiella}

Klebsiella were isolated and identified by culturing the clinical samples onto MacConkey agar plate where large mucoid colonies were selected. The selected colonies were further confirmed by culturing on Eosine Methylene Blue (EMB) agar plate. Then the presumptively identified Klebsiella colonies were checked for their Gram staining reaction.

\section{Biochemical characterization}

Biochemical tests employed were IMViC (Indole, Methyl red, Voges Proskeaur, Citrate), fermentation of sugars, oxidase and catalase test. For biochemical tests standard procedures were used (7).

\section{Antibiotic susceptibility test}

Susceptibility of bacterial isolates to different antibacterial agents was determined on Mueller-Hinton agar by modified Kirby-Bauer disc diffusion technique. The 0.5 McFarland standard isolates were inoculated with sterile cotton swab onto Mueller Hinton agar to make a lawn of bacterial growth. The plates were incubated overnight at $37^{\circ} \mathrm{C}$ and zone of inhibition were measured according to the criteria suggested by NCCLS, 2000 (27). Commercially available antimicrobial discs (Hi-Media Laboratories Pvt. Limited, India) that were used included Ampicillin $(25 \mu \mathrm{g})$, Amoxicillin-clavulanic acid $(30 \mu \mathrm{g})$, Ciprofloxacin $(05 \mu \mathrm{g})$, Co-trimoxazole $(25 \mu \mathrm{g})$, Ceftriaxone $(30 \mu \mathrm{g})$, Gentamicin $(10 \mu \mathrm{g})$, Tetracycline $(30 \mu \mathrm{g})$ and Nalidixic acid $(30 \mu \mathrm{g})$.

\section{Detection of ESBLS}

Isolates that exhibited reduced susceptibility to ceftriaxone $(30 \mu \mathrm{g})$ were considered as potential producers of ESBL (27). ESBL production in Klebsiella spp. was confirmed by double disc synergy test as described by Jarlier, 1988 (17). An inoculum of 0.5 Mcfarland standard was inoculated with sterile cotton swab onto Mueller Hinton agar to make a lawn of bacterial growth. $30 \mu \mathrm{g}$ disc of Ceftriaxone (30 $\mu \mathrm{g}$ ) was placed on the agar plate at a distance of $20 \mathrm{~mm}$ centre to centre from a Amoxicillin-clavulanic acid (20/10 $\mu \mathrm{g})$ disc prior to incubation. The plate was incubated overnight at $37^{\circ} \mathrm{C}$. Enhancement of the zone of inhibition of Ceftrioxone disc toward Amoxicillin-clavulanic acid confirmed the presence of extended-spectrum $\beta$-lactamases.

\section{Statistical analysis}

Analysis was performed by using Statistical Package for Social Science (SPSS Version 16) software and Excel Office program for the statistical analysis of this study. The t-test was done as a test of significance.

\section{RESULTS}

\section{Prevalence of Klebsiella (K. pneumonae and K. oxytoca)}

Out of 500 samples from clinical patients, Klebsiella spp. were reported in 120 cases through standard cultural and biochemical tests. Out of these 120 Klebsiella spp. 108 were K. pneumoniae and 12 were found $K$. oxytoca based on indole test. The prevalence rate of Klebsiella was about $24 \%$ among which $K$. pneumoniae accounted in $21.6 \%$ cases and $K$. oxytoca in $2.4 \%$ cases (Fig. 1 ).

Highest prevalence was observed in case of urine sample (66\%), followed by swab in $17 \%$ cases, pus in $10 \%$ cases and sputum in $7 \%$ cases (Fig. 2). Males were more affected (57\%) with Klebsiella spp. than females (43\%) (Fig. 3).

Persons older than 50 years irrespective of gender were more affected with Klebsiella spp., 58\% in male and 36\% 


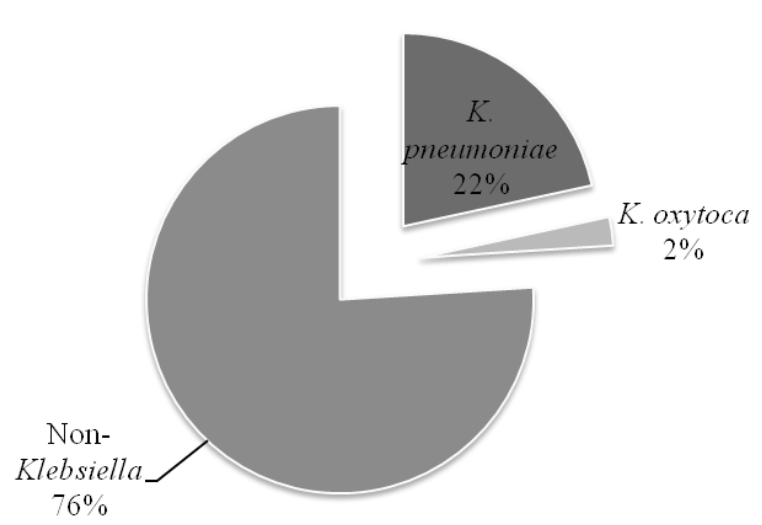

Fig. 1. Prevalence rate of $K$. pneumoniae and $K$. oxytoca among clinical samples.

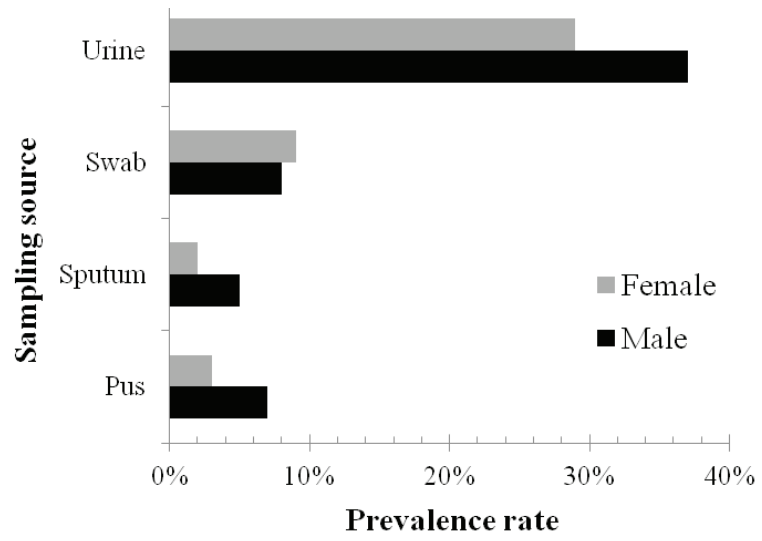

Fig. 2. Occurrences of Klebsiella spp. in different clinical samples.

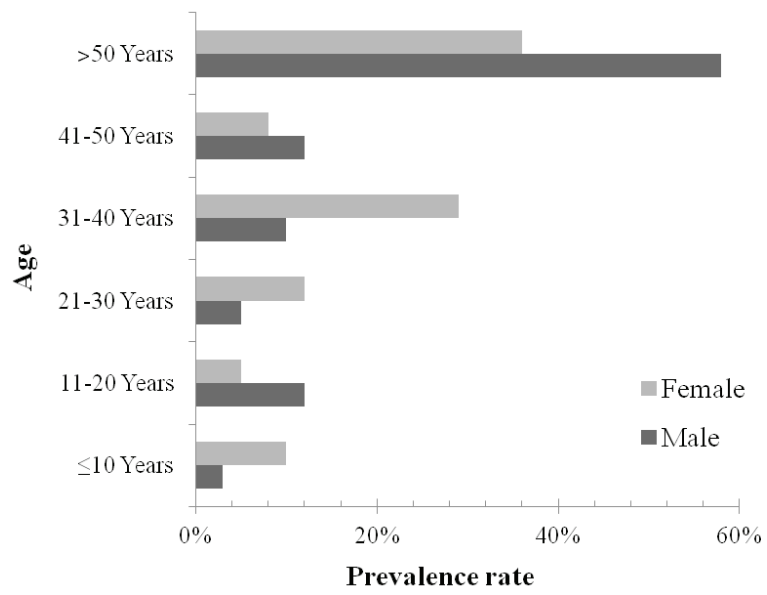

Fig. 3. Occurrences of Klebsiella spp. among different age groups.

in case of female (Fig. 3). The occurrence of infection due to Klebsiella spp. was found almost equivalent throughout the 12 months with some inclination in the months of June (12\% for male and $19 \%$ for female) and July (16\% for male and $19 \%$ for female) (Fig. 4).

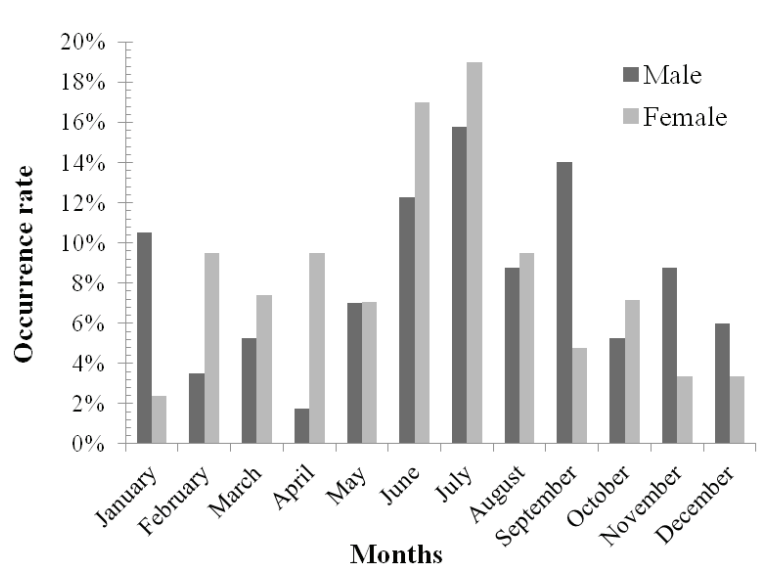

Fig. 4. Monthly occurrence rate of Klebsiella spp.

\section{Antibitic sensitivity pattern of Klebsiella}

The resistance pattern of Klebsiella isolates to Ampicillin, Amoxicillin, Ceftriaxone, Ciprofloxacin, Co-trimoxazole, Gentamicin, Nalidixic acid, Tetracycline was $100 \%, 90 \%, 45 \%, 40 \%, 45 \%, 25 \%, 50 \%$, 35\% respectively (Fig. 5). Highest sensitivity was observed against Gentamicin (75\%) followed by Tetracycline (65\%). Multidrug resistance (MDR) was observed in $55 \%$ of Klebsiella isolates where 12 isolates out of 120 were found resistant against all the antibiotics tested.

Antibiogram of $K$. pneumoniae revealed resistance to Ampicillin, Amoxicillin, Ceftriaxone, Ciprofloxacin, Cotrimoxazole, Gentamicin, Nalidixic acid, Tetracycline by $100 \%, 94 \%, 50 \%, 37.5 \%, 44 \%, 31 \%, 44 \%$ and $31 \%$ respectively (Fig. 6). Antibiotics that were found most potent against $K$. pneumoniae was Gentamicin and Tetracycline (69\% in both cases) followed by Ciprofloxacin (62.5\%). Multidrug resistance was observed among 56\% $K$. pneumoniae isolates.

Our data showed that $K$. oxytoca was $100 \%$ resistant towards Ampicillin which was similar to $K$. pneuminiae. However, unlike $K$. pneumoniae, $K$. oxytoca revealed resistance to Amoxicillin, Ceftriaxone, Ciprofloxacin, Co-

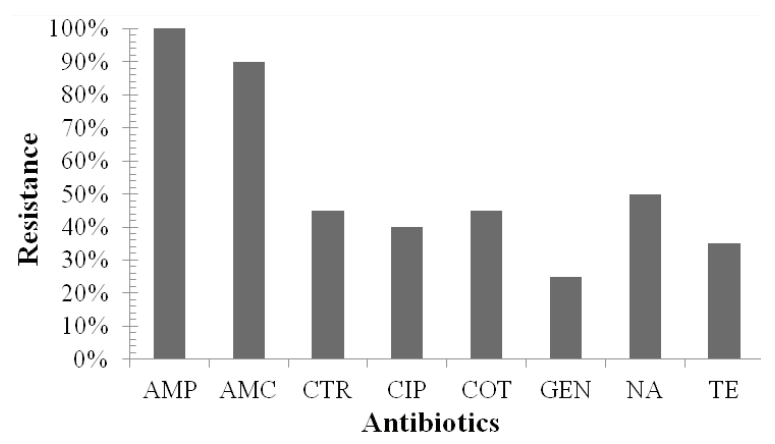

Fig. 5. Antibiotic resistance pattern of Klebsiella isolates. 


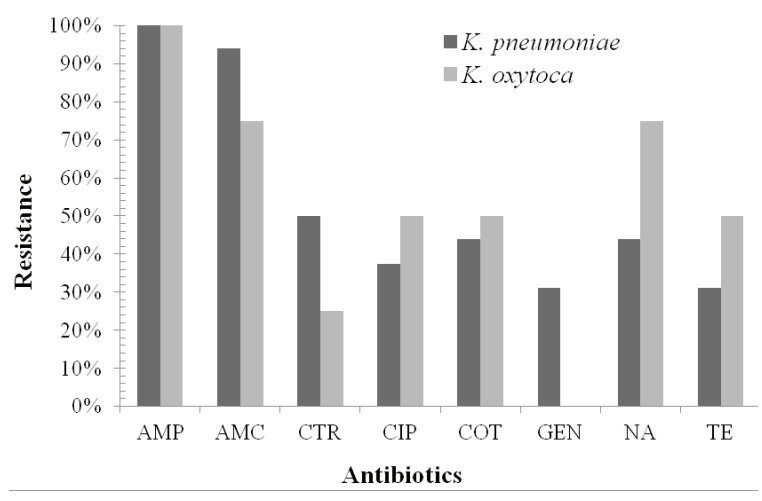

Fig. 6. Comparative antibiotic resistance pattern of $K$. pneumoniae and K. oxytoca.

trimoxazole, Gentamicin, Nalidixic acid, Tetracycline by $75 \%, 25 \%, 50 \%, 50 \%, 0 \%, 75 \%$ and $50 \%$ respectively (Fig. 6). Gentamicin was found to be the most potential drug followed by Ceftrioxone. Multidrug resistance was observed among $50 \% \mathrm{~K}$. oxytoca isolates.

\section{Potential ESBL among Klebsiella spp.}

Primarily 65\% of the Klebsiella isolates were identified as potential ESBL producer through the critical zone diameter concept, among which $69 \%$ were $K$. pneumoniae and $50 \%$ were $K$. oxytoca. However, the double disc synergy test revealed that $45 \%$ Klebsiella isolates possess ESBL property among which $K$. pneumoniae were 50\% and $K$. oxytoca were $25 \%$.

\section{DISCUSSION}

The high prevalence rate of Klebsiella among clinical patients has become a global concerns in recent years. In the present study, $24 \%$ prevalence rate of Klebsiella was observed among clinical patients from north eastern part of Bangladesh which is alarming since less prevalence rate (19.72\%) was observed previously in south eastern part of Bangladesh by Akter et al., 2014 (3). This difference in prevalence rate may be acceptable because the prevalence of Klebsiella infections varies in different geographical locations (33). The two clinically relevant species, K. pneumoniae and $K$. oxytoca was differentiated by the ability to produce indole from tryptophan (21). Majority of the isolates in the present study proved to be $K$. pneumoniae $(90 \%)$ which is in good agreement with previous studies $(12,38)$. For both organisms, however, prevalence was higher in males than in the female samples. Female in the age group of 31-40 years and greater than 50 years were found to be very much prone to Klebsiella associated infection whereas male has highest incidence of infection on age group greater than 50 years. Similar results have been previously reported by several investigators in Bangladesh who reported that men and women of elderly group were found to be very much prone to Klebsiella infection $(3,20)$. In Pakistan, males of older age and females of reproductive age were more prone to Klebsiella infections particularly by ESBL producers (33). Most of the Klebsiella were recovered from urine samples irrespective of age and gender, followed by swab, sputum and pus. Akter et al., 2014 (3) and Riaz et al., 2012 (33) also reported that urine is the principal source of Klebsiella.

Our data suggest that rates of Klebsiella incidence reach to the peak in the month of June and July, which are the two most humid and warmest months in Bangladesh. Such findings have been also observed by Anderson et al., 2008 (4) who demonstrated that environmental pressure during the warmest months may lead to an increase in Klebsiella infection. Characteristics of Klebsiella might have some role on such seasonal variation. Klebsiella are the most heat tolerant among all enteric pathogens (29) having maximal specific growth rate at $37^{\circ} \mathrm{C}$ (9). In addition, they are believed to survive at higher humidity (36).

The effectiveness of currently available antibiotics is decreasing due to the increasing number of resistant strains causing infections (26). Available therapeutic options for antibiotic-resistant organisms are severely limited, as these organisms frequently display a multidrug-resistant (MDR) phenotype $(19,23,31)$. In the present study, $K$. pneumoniae and $K$. oxytoca demonstrated varying degree of sensitivity pattern to different antibiotics. Most of the Klebsiella isolates showed resistance to the first line antibiotics that are commonly prescribed by the physicians. Our data showed that all the $K$. pneumoniae isolates were resistant to Ampicillin. Moderate level sensitivity were observed against Ceftriaxone, Ciprofloxacin, Co-trimoxazole, Gentamicin, Nalidixic acid and Tetracycline among which Gentamicin and Tetracycline showed good promise. Gentamicin like aminoglycosides have a good record against clinically important Gram-negative bacilli (10). Recently in India and Pakistan, about 16.70\% and $17.39 \%$ Klebsiella isolates were found sensitive against Gentamicin (32, 37). The difference among our data and the previously found lower sensitivity against Gentamicin may be due to selective pressure in different regions (22).

Emergence of multidrug resistant Klebsiella has become a major public health concern worldwide and has been associated with outbreaks of hospitalized infections in developing countries like Bangladesh due to the indiscriminate use of antibiotics (3). In the present study, multidrug resistance was found more common in $K$. pneumoniae (56\%) than in K. oxytoca (50\%). Various degrees of multidrug resistance among Klebsiella have been reported in different parts of the world. In Bangladesh, about $87 \%$ multidrug resistant Klebsiella were recorded (20) whereas in India and Pakistan, 54\% and 71.73\% Klebsiella isolates were reported to harbor multidrug resistance $(35,37)$. Chikwendu et al., 2010 in Nigeria reported $75.8 \%$ multidrug resistant Klebsiella isolates (6). Most recently, Gundogan and Avci, 2013, reported that 31.7\% Klebsiella isolates possess multidrug resistance (11). 
ESBL producing Klebsiella pose great challenges for efficient treatment of infections which requires new antibacterial compounds every now and then. Our study focused on the present status of ESBL producing Klebsiella. Interestingly all the ESBL producing Klebsiella isolates were found to be multidrug resistant, showing $100 \%$ resistance to Ampicillin, Amoxicillin, Ceftriaxone and Ciprofloxacin indicating relationship between multidrug resistance and ESBL possession. However, $12.5 \%$ non-ESBL K. pneumoniae possess multidrug resistance property while 33\% of the non-ESBL $K$. oxytoca harbour multidrug resistance.

The prevalence rate of ESBL producing Klebsiella is very high (45\%) in our study as compared to previous studies such as $40 \%$ in France (5) and 17\% in Pakistan (33). However, the present result for the occurrences of ESBL among Klebsiella isolates is significantly better than previous studies by Sharma et al., 2013 (34) and Jain and Mondal, 2007 (15) who found much higher rate $(67.04 \%$ and $58 \%$, respectively). ESBL producing $K$. pneumoniae (50\%) were found more prominent than K. oxytoca (25\%). In case of ESBL producing $K$. oxytoca, our result is in good agreement with the studies by Gundogan and Avci, 2013, who found that $26 \% K$. oxytoca were ESBL producer (11). However, in case of ESBL producing $K$. pneumoniae, the rate is much higher than found by Gundogan and Avci, 2013 (38.5\%) (11). Such high rate of ESBL among $K$. pneumoniae might be due to inappropriate use of antibiotics and self-medication against $K$. pneumoniae infection. However, our results are significantly better for ESBL producing $K$. pneumoniae than found by Afifi (2013) in Egypt (56.25\%), Jain et al. (2003) in India (86.6\%) and Ullah et al. (2009) in Pakistan (58.5\%) $(1,16,37)$.

The high frequencies with which antibiotics are used empirically to treat various diseases in Bangladesh suggest that there might be high rate of failure associated with eradication of microbial infections. Our results suggest that there is high antibiotic resistance towards commonly prescribed antibiotics and ESBLs rates among clinical Klebsiella isolates in the specific region of the country. Further studies need to be carried out from other parts of Bangladesh considering multidrug resistance and ESBL production rate among clinical and non clinical Klebsiella isolates. Continuous monitoring of ESBL, strict antibiotic policy along with conventional antibiogram will have a great impact in reducing bacterial resistance towards antibiotics and development of proper treatment options against Klebsiella infections.

\section{REFERENCES}

1. AFIFI M M 2013 Detection of extended spectrum beta-lactamase producing Klebsiella pneumoniae and Escherichia coli of environmental surfaces at upper Egypt. Int J Biol Chem 7: 58-68 http://dx.doi.org/10.3923/ijbc.2013.58.68

2. AGRAWAL P, GHOSH A N, KUMAR S, BASU B, KAPILA K 2008 Prevalence of extended-spectrum $\beta$-lactamases among Escherichia coli and Klebsiella pneumoniae isolates in a tertiary care hospital. Indian J Pathol Microbiol 51: 139-142
3. AKTER J, CHOWDHURY A M M A, FORKAN M A 2014 Study on prevalence and antibiotic resistance pattern of Klebsiella isolated from clinical samples in south east region of Bangladesh. Am J Drug Disc Dev 4: 73-79 http://dx.doi.org/10.3923/ajdd.2014.73.79

4. ANDERSON D J, HERVÉ R, CHEN L F, SPELMAN D W, HUNG Y-J, HUANG A T, SEXTON D J, RAOULT D 2008 Seasonal variation in Klebsiella pneumoniae bloodstream infection on 4 continents. J Infect Dis 197: 752-756

5. BRANGER C, LESIMPLE A L, BRUNEU B, BERRY P, ZECHOVSKY N L 1998 Long term investigation of clonal dissemination of $K$. pneumoniae isolates producing extended spectrum $\beta$-lactamases in a university hospital. J Med Microbiol 47: 201-209

6. CHIKWENDU C I, AMADI E S, OBI R K 2010 Prevalence and antimicrobial resistance in Pseudomonas aeruginosa and Klebsiella pneumoniae isolates from non-clinical urine samples. $N$ Y Sci J 3: 194-200

7. CRUICKSHANK R 1980 Medical Microbiology. 12th eds. Edinburg: Churchill Livingstone, UK, p 170-189

8. CHOU H, LEE C Z, MA L C, FANG C T, CHANG S C, WANG J T 2004 Isolation of a chromosomal region of Klebsiella pneumonia associated with allantoin metabolism and liver infection. Infect Immun 72: 3783-3792

http://dx.doi.org/10.1128/IAI.72.7.3783-3792.2004

9. ESENER A A, ROELS J A, KOSSEN N W F 1981 The influence of temperature on the maximum specific growth rate of Klebsiella pneumoniae. Biotechnol Bioengineering 23: 1401-1405 http://dx.doi.org/10.1002/bit.260230620

10. GONZALEZ L S, SPENCER J P 1998 Aminoglycosides: a practical review. Am Fam Physician 58: 1811-1820

11. GUNDOGAN N, AVCI E 2013 Prevalence and antibiotic resistance of extended-spectrum beta-lactamase (ESBL) producing Escherichia coli and Klebsiella species isolated from foods of animal origin in Turkey. Afr J Microbiol Res 7: 4059-4064

12. HANSEN D S, GOTTSCHAU A, KOLMOS H J 1998 Epidemiology of Klebsiella bacteraemia: a case control study using Escherichia coli bacteraemia as control. J Hosp Infect 38: 119-132

13. HANSEN D S, AUCKEN H M, ABIOLA T, PODSCHUN R 2004 Recommended test panel for differentiation of Klebsiella species on the basis of a trilateral interlaboratory evaluation of 18 biochemical tests. J Clin Microbiol 42: 3665-3669

14. HORAN T, CULVER D, JARVIS W, EMORI G, BANERJEE S, MARTONE W, THORNSBERRY C 1988 Pathogens causing nosocomial infections. Antimicrob Newslett 5: 65-67 http://dx.doi.org/10.1016/0738-1751(88)90027-5

15. JAIN A, MONDAL R 2007 Prevalence and antimicrobial resistance pattern of extended spectrum beta-lactamase producing Klebsiella spp isolated from cases of neonatal septicaemia. Indian J Med Res 125: 89-94

16. JAIN A, ROY I, GUPTA M K, KUMAR M, AGARWAL S K 2003 Prevalence of extended-spectrum beta-lactamase-producing Gram-negative bacteria in septicemia neonates in a tertiary care hospital. J Med Microbiol 52: 42-45

17. JARLIER V, NICOLAS M H, FOURNIER G, PHILIPPON A 1988 Extended spectrum $\beta$-lactamases conferring transferable resistance to newer $\beta$-lactam agents in Enterobacteriaceae: Hospital prevalence and susceptibility patterns. Rev Infect Dis 10: 867-878

18. LEE S O, LEE E S, PARK S Y, KIM S Y, SEO Y H, CHO Y K 2004 Reduced use of third-generation cephalosporins decreases the acquisition of extended spectrum $\beta$-lactamases producing Klebsiella pneumoniae. Infect Control Hosp Epidemiol 25: 832-837 http://dx.doi.org/10.1086/502304

19. LEWIS J S, HERRAERA M, WICKES B, PATTERSON J E, JORGENSEN J H 2007 First report of the emergence of CTX-Mtype extended-spectrum $\beta$-lactamases (ESBLs) as the predominant 
ESBL isolated in a U.S. healthcare system. Antimicrob Agents Chemother 51: 4015-4021

20. LINA T T, RAHMAN S R, GOMES D J 2007 Multiple antibiotic-resistance mediated plasmids and integrons in uropathogenic Escherichia coli and Klebsiella pneumoniae. J Microbiol 24: 19-23

21. MASLOW J N, BRECHER S M, ADAMS K, DURBIN S A, LORING S, ARBEIT R D 1993 Relationship between indole production and differentiation of Klebsiella species: indole-positive and -negative isolates of Klebsiella determined to be clonal. J Clin Microbiol 31: 2000-2003

22. MILLER G H, SABATELLI F J 1997 The most frequent aminoglycoside resistance mechanisms--changes with time and geographic area: a reflection of aminoglycoside usage patterns? Aminoglycoside Resistance Study Groups. Clin Infect Dis 24 (suppl 1): S46-S62 http://dx.doi.org/10.1093/clinids/24.Supplement_1.S46

23. MOLAND E S, HANSON N D, BLACK J A, HOSSAIN A, SONG W, THOMSON K S 2006 Prevalence of newer $\beta$-lactamases in gram-negative clinical isolates collected in the United States from 2001 to 2002. J Clin Microbiol 44: 3318-3324

24. MSHANA S E, KAMUGISHA E, MIRAMBO M, CHAKRABORTY T, LYAMUYA E 2009 Prevalence of multiresistant gram-negative organisms in a tertiary hospital in Mwanza, Tanzania. BMC Res Notes 2: 49

25. NAUMOVSKI L, QUINN J P, MIYASHIRO D, PATEL M, BUSH K, SINGER S B, GRAVES D, PALZKILL T, ARVIN A M 1992 Outbreak of ceftazidime resistance due to a novel extended-spectrum beta-lactamase in isolates from cancer patients. Antimicrob Agents Chemother 36: 1991-1996

26. NAWAZ S K, RIAZ S, HASNAIN S 2009 Screening for antimethicillin resistant Staphylococcus aureus (MRSA) bacteriocin producing bacteria. Afr J Biotech 8: 365-368

27. NCCLS 2000 Methods for Dilution Antimicrobial Susceptibility Tests for Bacteria that Grow Aerobically. 5th Edn, National Committee for Clinical Laboratory Standards, Approved Standard M7A5, Wayne, PA, USA

28. O'BRIEN T F 1992 Global surveillance of antibiotic resistance. $N$ Engl J Med 326: 339-340 http://dx.doi.org/10.1056/NEJM199201303260510
29. OKADA S, GORDON D M 2001 Host and geographical factors influence the thermal niche of enteric bacteria isolated from native Australian mammals. Mol Ecol 10: 2499-2513 http://dx.doi.org/10.1046/j.0962-1083.2001.01384.x

30. OKEKE I N, LAMIKANRA A, EDELMAN R 1999 Socioeconomic and behavioral factors leading to acquired bacterial resistance to antibiotic in developing countries. Emerg Infect Dis 5: $18-27$

31. OKONKO I O, SOLEYE F A, AMUSAN T A, OGUN A A, OGUNNUSI T A, EJEMBI J 2009 Incidence of multi-drug resistance (MDR) organisms in Abeokuta, Southwestern Nigeria. Global J Pharmacol 3: 69-80

32. REVATHI G, PURI J 1998 Bacteriology of burns. Burns 24: 347349 http://dx.doi.org/10.1016/S0305-4179(98)00009-6

33. RIAZ S, FAISAL M, HASNAIN S 2012 Prevalence and comparison of Beta-lactamase producing Escherichia coli and Klebsiella spp from clinical and environmental sources in Lahore, Pakistan. Afr J Microbiol Res 6: 465-470

34. SHARMA M, PATHAK S, SRIVASTAVA P 2013 Prevalence and antibiogram of extended-spectrum $\beta$-lactamase (ESBL) producing Gram-negative bacilli and further molecular characterization of ESBL producing Escherichia coli and Klebsiella spp. J Clin Diag Res 7: 2173-2177 http://dx.doi.org/10.7860/jcdr/2013/6460.3462

35. SIKARWAR A S, BATRA H V 2011 Prevalence of antimicrobial drug resistance of Klebsiella pneumoniae in India. Int J Biosci Biochem Bioinform 1: 211-215 http://dx.doi.org/10.7763/IJBBB.2011.V1.38

36. THEUNISSEN H J, LEMMENS-DEN TOOM N A, BURGGRAAF A, STOLZ E, MICHEL M F 1993 Influence of temperature and relative humidity on the survival of Chlamydia pneumoniae in aerosols. Appl Environ Microbiol 59: 2589-2593

37. ULLAH F, MALLIK S A, AHMED J 2009 Antimicrobial susceptibility pattern in Klebsiella pneumonia from urinary tract infections in North-West of Pakistan. Afr J Microbiol 3: 676-680

38. WATANAKUNAKORN C, JURA J 1991 Klebsiella bacteremia: a review of 196 episodes during a decade (1980-1989). Scand J Infect Dis 23: 399-405 http://dx.doi.org/10.3109/00365549109075086 